\title{
Designing innovative food products for correcting adaptive immunity and increasing the body's resistance to infectious diseases
}

\author{
Ludmila Eliseeva ${ }^{1}$, Daria Kokorina $^{1}$, Yuriy Belkin ${ }^{1}$, Elena Zhirkova $^{1}$, and Vladimir \\ Orobets $^{2}$ \\ ${ }^{1}$ Plekhanov Russian University of Economics, Stremyanny lane, 36, 117997 Moscow, Russia \\ ${ }^{2}$ Stavropol State Agrarian University, Zootekhnichesky Lane, 355017 Stavropol, Russia
}

\begin{abstract}
The analysis of the relationship between the nutrition structure and the immune status of a person has been carried out. The role of nutrition in preventing alimentary-dependent and infectious diseases is considered. The nutritional and biological value of quinoa pseudo-grain culture has been studied. A recipe and technology for enriching wheat bread with functional and immunomodulating ingredients of quinoa has been developed. The content of functional ingredients in fortified bread has been determined. From laboratory animal testing, it was found that wheat bread enriched with functional ingredients of quinoa flour reduces blood cholesterol, low density lipoproteins, and helps to reduce blood sugar. The results obtained make it possible to recommend these products for strengthening adaptive immunity and for prevention of infectious diseases of diabetes mellitus.
\end{abstract}

\section{Introduction}

Across the lifespan, a person lives in an environment where they are constantly exposed to various risks of microbiological and chemical nature. In recent years, the risk of coronavirus infection has posed a great danger. What is essential for the prevention of infectious diseases is the level of innate and adaptive immunity of the body, which is formed immediately after the birth of a child and undergoes various qualitative and quantitative changes throughout life. It has been proven that the immune system includes various mechanisms of the immune response, which are aimed at protecting the body from viral, bacterial, fungal infections by synthesizing specific antibodies of protein nature. The main task of such antibodies is to recognize and destroy foreign organisms. The functional activity level of the immune system depends on the physiological state of the main body systems.

Over the past twenty years, international research and medical practice have been studying the relationship between nutrition structure and the body's immune status. It was found that, along with pharmacological medications, nutrition plays an indisputably important role while forming adaptive immunity. To ensure correct eating behavior, there should be a balanced intake of not only the established ratio of proteins, fats, carbohydrates, but also the quantitative combination of nutrients within each group. Nutrition and 
immunity are closely related to each other, because healthy eating habits provide metabolism with all the necessary elements to construct the chemical compounds involved in the formation of immune responses [5,7]. For example, a lack of protein reduces resistance to infectious diseases, because it is required for the formation of antibodies and leukocytes, which are important elements of immune responses. They consist of protein and contain amino acids that are unable to be synthesized by the body. Such amino acids come only with food, and the most important ones are lysine and threonine. Lysine is directly involved in the synthesis of antibodies. Threonine, in addition to participating in the construction of antibodies, takes an active part in resisting stress and normalizes the activity of the nervous system, which is also important for the formation of stable immunity. In terms of providing balanced diet the WHO also pays special attention to the content of biologically active compounds, including vitamins, minerals, antioxidants, etc., which have a direct effect on the regulation of the physiological activity of the body and affects the immune status. There are especially high risks to lack individual biologically active compounds for children. For example, according to the WHO, dysmicroelementosis and deficiency of certain vitamins sharply reduce immunity and can lead to death. Deficiency of zinc and vitamin $\mathrm{A}$ in children under age 5 has led to the development of infectious diseases, which has been fatal among almost a quarter of patients $[1,2]$.

Nutrition surveillance in Russia has shown an imbalance in the nutrition structure. Deficiency of vitamins and microelements was revealed. For example, vitamin D deficiency can occur even in the southern regions of Russia. Shortage of vitamin $\mathrm{C}$ is detected in 38-90\% of students, vitamin B2 - in 35-75\%, carotene - in $84 \%$. But the timely correction of the deficit helps to increase the adaptive immunity and the quality children's life. In this regard, comprehensive research is being carried out in the field of dietetics and nutritionology, which makes it possible to scientifically substantiate the corrective effect of the nutritional structure on the immune status of the body and determine the regulatory functions of certain types of biologically active food components. Currently, the immunotropic effect of a number of vitamins, minerals, amino acids, Omega -3 fatty acids, antioxidants and their role in the prevention of infectious diseases has been proven. Covering the deficiency of immunocorrecting macro- and micronutrients leads to the activation of the body's immune response and an increase in the body's resistance to infectious diseases [2,7]. Vitamins $C$, groups B, D, E and $\beta$-carotene are recognized as indisputable immunocorrectors. They prevent free radical oxidative processes in the cell, protect against membrane destruction. Recent research confirms the antiviral effect of vitamin $\mathrm{D}$. It has been found that vitamin $\mathrm{E}$ exhibits a nonspecific immunoregulatory function, stimulates the synthesis of interleukin-2, leukocytes, inhibits suppressive activity and activates the activity of the main elements of the immune system. Vitamin $\mathrm{C}$ is involved in the synthesis of thyroid hormones and corticosteroid hormones, which are necessary to increase the body's adaptation to adverse conditions and infectious diseases. Vitamin $\mathrm{C}$ regulates the activity of lymphocytes and activates the synthesis of interferon. Moreover, vitamin $\mathrm{C}$ is a synergist of vitamin $\mathrm{E}$ and protects it from free radicals. Vitamin $\mathrm{A}$ is involved in the synthesis of antibodies. Vitamin A deficiency in children is considered to be a risk factor for fatal cases in infectious diseases. In international medical practice, there is data on the immunomodulatory properties of B vitamins, which are actively involved in cellular metabolism. The vitamins riboflavin, pyridoxine, folic acid and pantothenic acids are directly involved in the synthesis of antibodies. The immunomodulatory properties of a number of microelements have been confirmed, incl. zinc and selenium, which are also involved in the synthesis of antibodies. Of particular importance are dietary fiber and probiotics, which regulate and normalize the activity of the intestinal microflora. 
In Russia, according to the results of monitoring, which is regularly carried out under the guidance of the Federal Research Centre for Nutrition and Biotechnology, the level of deficiency of complete proteins, Omega-3 fatty acids, dietary fiber of a complex of vitamins and minerals has been established. The most effective way to rehabilitate the health of the population, especially children, is the correction of the immune status by enriching the diet with deficient nutrients $[11,12]$. In the international and domestic practice of the healthy food production, the most acceptable and effective way to replenish the deficient macro- and micronutrients is considered to be the production of food fortified with the deficient nutrients for mass consumption and daily demand. In addition, such food should be available for all population groups.

Bread is one of the most popular mass-produced products of daily demand. Therefore, it is bakery products that are used as the basis for enriching the diet with limited ingredients. According to BusinesStat, sales of bakery products in Russia have declined by more than $5 \%$ over the past five-year period. In 2020, 6.2 million tonnes were produced, while production in 2019 was 6.3 million tonnes. At the same time, there is a steady trend towards an increase in long-term storage products, fortified and specialized bakery products. According to academician V. A. Tutelyan, the number of consumers who consciously purchase products for healthy eating, incl. fortified bakery products, increases annually. Currently, the market for fortified bakery products is about $1 \%$. This volume along with the assortment do not satisfy the constantly growing demand of consumers. As provided by the Concept for the Development of Specialized and Functional Bakery in the Russian Federation (Bread is Health), the task was set to reduce nutritional deficiencies in the population's diet through the production of fortified bakery products, the volume of which should increase to $5 \%$ in the area of the domestic food industry $[7,8,12,13]$.

The assortment of fortified bakery products is divided into 2 large groups depending on the method of fortification: products made with premixes (vitamin and mineral complexes) and products made of natural raw ingredients of plant and animal origin. Consumers prefer bread enriched with natural raw functional ingredients. The most common is the modeling of the recipe composition of bread by replacing a part of the flour with raw ingredients of plant origin, taking into account the correction of the chemical composition of the finished one in accordance with the physiological norms of consumption [13]. To obtain a wheat bread balanced in nutritional and biological value, it is necessary to use a raw source that increases the content of protein, compensates for the limiting essential amino acids, and increases the content of polyunsaturated fatty acids, dietary fiber, vitamins and minerals. All these requirements are met by the chemical composition and consumer characteristics of the seeds of the quinoa pseudo-grain culture, which is grown in more than 70 countries of the world. In Russia, breeding work on the introduction of the crop has been carried out since 2009 and in 2017 the Ministry of Agriculture included quinoa in the state register of breeding achievements approved for use. Currently, an active development and production is being established in the Krasnodar Krai, in the Central Black Earth Region and even in the Moscow region. The UN FAO views quinoa as a promising source of essential macroand micronutrients that can be widely used to address the global food security problem. The UN General Assembly proclaimed 2013 the "International Year of Quinoa" [8,9,16]. Studies of foreign and domestic scientists confirm the unique nutritional value of the culture of quinoa $[3,4,6]$.

The purpose of the research at the first stage of the work was to carry out a comparative analysis of the chemical composition of 4 types of quinoa imported to the food market in Russia and quinoa grown in the Moscow region to justify the choice of the type of quinoa for enrichment of wheat bread. At the second stage of the work, using the developed computer program, the design of the fortified bread recipe was carried out and the content of the main macro- and micronutrients, which can be considered as functional ingredients, 
was determined, and the fortified bread production technology was optimized. At the third stage, pre-clinical tests of fortified bread were carried out to confirm the possibility of its use in order to increase adaptive immunity. Based on the results of the chemical composition study, it was found that samples of test baked goods obtained from light varieties of quinoa seeds of the Trademark «Products of the XXII century» possessed the largest nutritional value, physicochemical quality indicators and organoleptic characteristics. Comparative characteristics of the nutritional value of the products are presented in table 1 .

Table 1. Comparative chemical composition of quinoa and soft wheat

\begin{tabular}{|c|c|c|c|}
\hline \multirow{2}{*}{ Main Factors } & \multicolumn{3}{|c|}{ Nutrient content, \% } \\
\cline { 2 - 4 } & $\begin{array}{c}\text { «Products of the XXII } \\
\text { century» quinoa }\end{array}$ & $\begin{array}{c}\text { Quinoa from the } \\
\text { Moscow region }\end{array}$ & Soft wheat \\
\hline Protein, g & 15,3 & 13,5 & 12,2 \\
\hline Fat, g & 5,7 & 5,8 & 2,0 \\
\hline Carbohydrates, g & 61,0 & 61,2 & 67,9 \\
\hline Fiber, g & 2,9 & 2,7 & 1,9 \\
\hline Ash, g & 2,5 & 2,4 & 1,8 \\
\hline
\end{tabular}

The composition of quinoa flour, recommended as a raw ingredient for the production of fortified wheat bread, differs from quinoa grown in the Moscow region and soft wheat in a higher content of protein, fat, fiber and ash elements. To determine the effectiveness of using quinoa for fortification of bread, the comparative amino acid composition of wheat and quinoa was studied. It was found that tryptophan, lysine and threonine are the limiting amino acids in the composition of wheat protein. In the composition of proteins of the studied quinoa species, the presence of limiting amino acids has not been established; for the amino acids lysine, threonine, methionine, and cysteine, the amino acid rate significantly exceeds $100 \%$, therefore, they can serve to compensate for the limiting amino acids in soft wheat flour. Also, the amino acid rate of BCAA in quinoa also exceeds by $120-150 \%$ the rate in soft wheat. When enriching food products for the correction of nutritional value and modulation of immunity, great importance is attached to the content of polyunsaturated fatty acids (PUFA) and specifically to Omega-3 acids. Quinoa has a fat content of 5.7\%. The total PUFA content was about $50 \%$ in quinoa and $23 \%$ in wheat flour. Quinoa was dominated by linoleic acid (45\%) and linolenic acid - 7.2\% of the total PUFA. Omega-3 fatty acid plays a great role in the regulation of metabolic processes in the body and strengthening the immune system. The recommended ratio of $\omega-6: \omega-3$ PUFA is in the range of 5-10:1. In quinoa seeds, this ratio is close to optimal and amounts to 5-7:10, therefore, when wheat bread is fortified with quinoa flour, the content of $\omega-3$ acids increases.

Considering the great importance attached to the enrichment of food with minerals, it was found that quinoa exceeds wheat in terms of $\mathrm{Zn}$ by 52 , Ca by $40, \mathrm{~K}$ by $50, \mathrm{Mg}$ by $55, \mathrm{P}$ by $20 \%$. In the studied objects, vitamins of group B and PP were determined. A significant excess of vitamins B3, B6, B9 and PP was found in quinoa flour and amounted to 110, 84, 44 and $170 \%$, respectively. Therefore, it can be assumed that in the production of bread, it is possible to achieve a correction of the chemical composition of fortified wheat bread according to the studied parameters to a level that meets the requirements for functional ingredients. 
When determining the maximum possible dose to fortify wheat bread with quinoa flour, it is necessary to take into account the effect of the dose of added quinoa flour on the functional and technological properties of the dough, considering the fact that quinoa flour does not contain gluten, but contains 5.7\% fat and 55-57\% starch. The revealed differences can have a significant impact on the dough preparation process and consumer characteristics of finished products. To optimize the bread recipe, test baking of samples was carried out with the replacement of wheat flour in the range of $10-25 \%$ with a step of $5 \%$. The analysis of the functional and technological characteristics of the dough and the organoleptic characteristics of the obtained samples made it possible to determine the recommended dose of quinoa flour in the recipe, which is $17 \%$. Analysis of the chemical composition of an enriched bread sample let establish the increment in nutritional value ( $\mathrm{g} /$ $100 \mathrm{~g})$ at: 2.2 in protein content; 1.5 - dietary fiber; 2.0 - fat. The content of essential amino acids has increased by $33 \%$ incl. lysine $-15 \%$, threonine $-16 \%$ and methionine $-24 \%$. A comparative analysis of the increase in macro-micronutrients is presented in Table 2 (the table contains data on nutrients, the content of which in fortified bread exceeds $15 \%$ of satisfying the daily requirement). The results obtained confirm the prospect of replacing $17 \%$ of wheat flour with quinoa flour to increase its nutritional value. Many functional ingredients recognized as nutrients that can correct the body's adaptive immunity complete protein, essential amino acids (lysine and threonine), $\omega-3$ fatty acids, dietary fiber, iron, magnesium, phosphorus and $\mathrm{B}$ vitamins satisfy the daily requirement of more than $15 \%$. Therefore, they can be considered as immunomodulators and functional ingredients $[3,4,17]$. To neutralize the negative effect of reducing the gluten content in the recipe mixture, the dough preparation technology has been optimized. To improve the baking properties of the dough, the methodological recommendations of the Research Institute of Bread Products were used. Additionally, $2 \%$ of dry gluten was added to the composition of the recipe, and a complex ferment of lactic acid bacteria (LAB) was introduced, which made it possible to increase the quantity and quality of gluten. When comparing different technologies for preparing dough, the advantage was given to the use of a steam-free method with LAB, which made it possible to improve the structural and mechanical characteristics of the dough, reduce the duration of fermentation in comparison with the traditional method and increase the dimensional stability, porosity, volumetric bread yield and organoleptic characteristics [18]. The design of the optimal bread recipe based on the obtained experimental results was carried out using the developed computer program.

Table 3. Nutritional value of control and fortified samples of bakery products

\begin{tabular}{|l|c|c|c|c|}
\hline Nutrient content & $\begin{array}{c}\text { Grade 1 } \\
\text { wheat flour } \\
\text { bread }\end{array}$ & $\begin{array}{c}\text { Bread with quinoa flour } \\
\text { and dry wheat gluten }\end{array}$ & $\begin{array}{c}\text { Daily } \\
\text { requirement, g }\end{array}$ & $\begin{array}{c}\text { Meeting the daily } \\
\text { requirement, \% }\end{array}$ \\
\hline Protein, g & 7,71 & 14,5 & 75 & 19,3 \\
\hline Dietary fiber, g & 3,45 & 5,0 & 30 & 16,7 \\
\hline Magnesium, g & 30,87 & 59,10 & 400 & 15,0 \\
\hline Phosphorus, g & 86,30 & 152,90 & 800 & 19,11 \\
\hline Iron, g & 1,51 & 2,91 & 14 & 20,8 \\
\hline Thiamine, g & 0,18 & 0,30 & 1,4 & 21,4 \\
\hline Riboflavin, g & 0,06 & 0,24 & 1,6 & 15,0 \\
\hline
\end{tabular}

* Average daily requirement for the basic nutrients and energy for the population 
To study the physiological effectiveness of enriched wheat bread with functional ingredients of quinoa, pre-clinical tests of the samples were carried out on white outbred rats in accordance with the approved methodology in vivo. The effect of fortified bread with an additive on the results of analyzes of biochemical, clinical parameters of blood, on the microbiota of the large intestine was studied, postprandial glycemia of laboratory animals and dynamics of body weight gain. White outbred rats were divided into 2 groups, 6 individuals in each group. The observation period for the animals lasted for 14 days in order to monitor their condition, behavior, food and water intake, the manifestation of symptoms of intoxication, and possible death. The study of postcranial glycemia was performed on the 14th day. To study the intestinal microflora, material sampling and microbiological studies were carried out in accordance with the methodological instructions 4.2.2039-05. The content of the main representatives of the intestinal microflora was determined: Escherichia, bifidobacteria and lactobacilli, opportunistic bacteria. The mentioned microorganisms were identified by their culture, morphological, tinctorial and biochemical properties. From the experiments on laboratory animals, it was found that wheat bread, enriched with the functional ingredients of quinoa flour, helps to reduce blood cholesterol, low density lipoproteins, and sugar content. The results obtained make it possible to recommend these products for strengthening adaptive immunity and for the prevention of infectious diseases and diabetes mellitus.

\section{References}

1. A. E. Bogomolov, S. V. Zaikov, Clinical Immunology. Allergology. Infectology, 2(61), 43 (2013)

2. V. M. Delyagin, Effective Pharmacotherapy, 16(34), 16 (2020)

3. L. G. Eliseeva, D. S. Kokorina, E. V. Zhirkova, Quality And Safety Of Goods: From Production To Consumption. Materials of the International Scientific and Practical Conference dedicated to the 60th anniversary of the revival of the Department of Commodity Science and Expertise of Goods, 199 (2019)

4. L. G. Eliseeva, E. V. Zhirkova, D. S. Kokorina, International Scientific And Practical Conference Dedicated To The Memory Of Gorbatov V. M., 1, 79 (2018)

5. I. A. Gromov, R. M. Torshkhoeva, A. A. Alekseeva, L. S. Namazova, Effective Pharmacotherapy, 1, 69 (2008)

6. L.E.A. James, Advances in Food and Nutrition Research, 58, 31 (2009)

7. V. M. Kodentsev, O. A. Vrzhesinskaya, D. V. Risnik, D. B. Nikityuk, V. A. Tutelyan, Problems Of Nutrition, 86(4), 13 (2017)

8. M.J. Koziol, Journal of Food Composition and Analysis, 5, 35 (1992)

9. E. A. Martínez, Estado del arte de la quinua en el mundo en 2013, 331 (2014)

10. T.V. Schekoldina, L.Ya. Rodionova, E.A. Chernikhovets, Scientific Journal of KubSAU, 121(07), 1 (2016)

11. V. B. Spirichev, Federal And Regional Aspects Of Healthy Eating Policy, 45 (2002)

12. V.B. Spirichev, L.N. Shatnyuk, Food Industry, 4, 20 (2010)

13. Bread and bakery products market in Russia 2020: revenue growth amid falling consumption, https://news.unipack.ru/

14. Ya.S. Petysh, Enrichment of bakery products with vitamins and minerals: global trends (2017) https://bake.ingredients.pro/ 
15. Quinoa International Year - 2013, Food and Agriculture Organization of the United Nations, http://www.fao.org

16. Master Plan for the International. Year of Quinoa, A Future Sown Thousands of Years Ago international year of quinoa, 26 (2013)

17. L.G. Eliseeva, E.V. Zhirkova, D.S. Kokorina, Yu.D. Belkin, N.A. Gribova, International Journal of Control and Automation, 13(1), 180 (2020)

18. L.G. Eliseeva, D.S. Kokorina, E.V. Zhirkova, S.A. Smirnova, E.V. Nevskaya, IOP Conference Series. Earth and Environmental Science, 670 (2021) 